\title{
INVESTIGACIÓN CIENTÍFICA Y TECNOLÓGICA
}

Cómo citar (APA): Campo Salazar, O. I. \& Montenegro Muñoz, M.I. (2018). Propuesta de un nuevo diseño de órtesis post-tratamiento de pie equino varo congénito. Revista Colombiana de Rehabilitación, 17 (2), 112-126 https://doi.org/10.30788/RevColReh.v17. n2.2018.338

Esta obra se encuentra bajo licencia internacional CC BY 4.0. 



\section{Introducción}

$\mathrm{E}$ $\mathrm{n}$ un estudio realizado por la Universidad del Valle y la Universidad ICESI en 2004 en la ciudad de Cali, se concluyó que de 32995 nacimientos (ocurridos durante los 4 años y 7 meses que duró el estudio), 735 presentaron un defecto congénito. De aquí se observa que en la ciudad de Cali hay una prevalencia de defectos congénitos de 2.22 por 10000 nacimientos. El estudio también reportó que los defectos con mayor prevalencia fueron la polidactilia y pie equino varo con 22.1 y 17.6 casos por 10000 nacimientos respectivamente (Pachajoa, Ariza, Isaza \& Méndez, 2011).

En otro estudio realizado por la Pontificia Universidad Javeriana de Bogotá, el cual fue enfocado en el estudio de la frecuencia de malformaciones congénitas haciendo una evaluación y pronóstico de 52744 nacimientos en tres ciudades colombianas, se obtuvo que 3.12 \% de esta población presentó alguna malformación congénita. Se encontró que el pie equino varo, la polidactilia y el labio y paladar hendidos, afectaron más a los pacientes de sexo masculino, con una diferencia estadísticamente significativa, ya que se encontró que la relación hombre/ mujer para defectos de las extremidades fue de 1.24 (Zarante, Franco, Lopez \& Fernández, 2010).

Dentro del estudio, anteriormente mencionado, se encontró que el pie equino varo tenía una prevalencia del 25.0 por 100oo. Se determinó que según la escala diseñada para determinar el pronóstico de las malformaciones congénitas, del total de las malformaciones, el 1.1\% pertenecen al grupo I (malformaciones mayores incompatibles con la vida), 54\% pertenecen al grupo II (malformaciones mayores que tienen un alto riesgo de mortalidad o discapacidad grave) y $44.8 \%$ pertenecen al grupo III (malformaciones compatibles con una vida normal) en el cual se encuentra el pie equino varo congénito (Zarante et al, 2010).

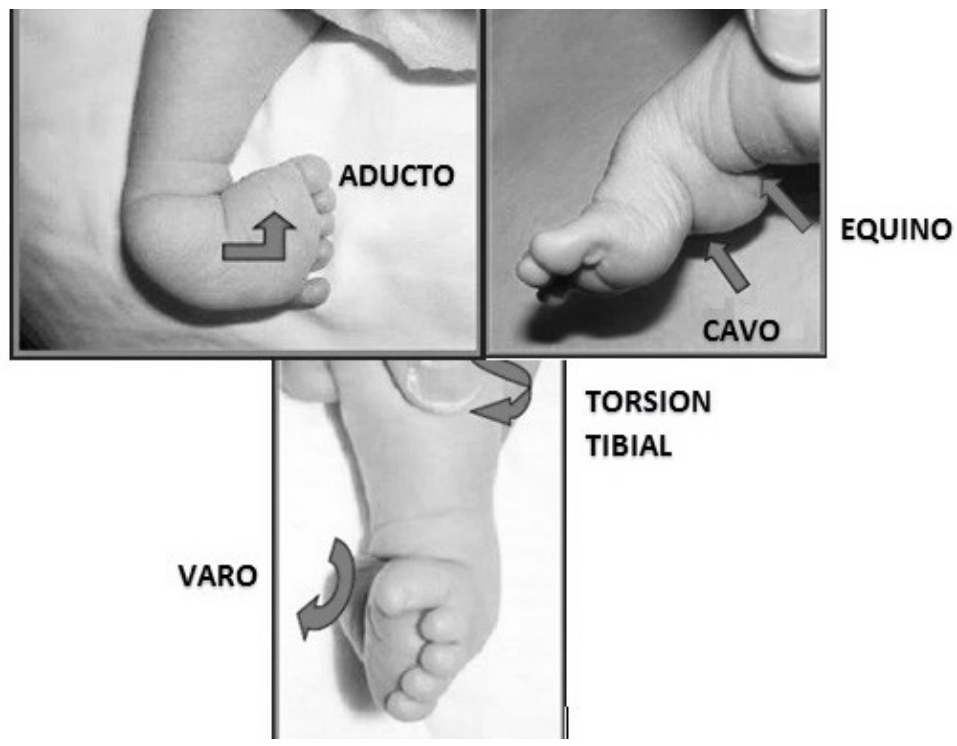

Figura 1. Morfología pie equino varo.

El pie equino varo consiste en una patología congénita en la cual, el individuo nace con una malformación en el pie que adopta una postura anómala ya que la punta del pie se dirige hacia abajo (equino) y la planta del pie se dirige hacia adentro (varo) (ver Figura 1). 
Para corregir esta deformidad, se emplea el método de Ponseti que consiste en la manipulación del pie por medio de la aplicación de una serie de yesos, que van redireccionando progresivamente el pie para alinearlo con la pierna y corregir la posición en varo. A este tratamiento le sigue una cirugía conocida como tenotomía, para lograr un alargamiento del tendón de Aquiles, lo que termina por corregir la postura en equino. Para terminar el tratamiento se utiliza una órtesis que consiste en dos botas unidas por una barra de metal, la cual mantiene el pie en abducción y en dorsiflexión (ver Figura 2).
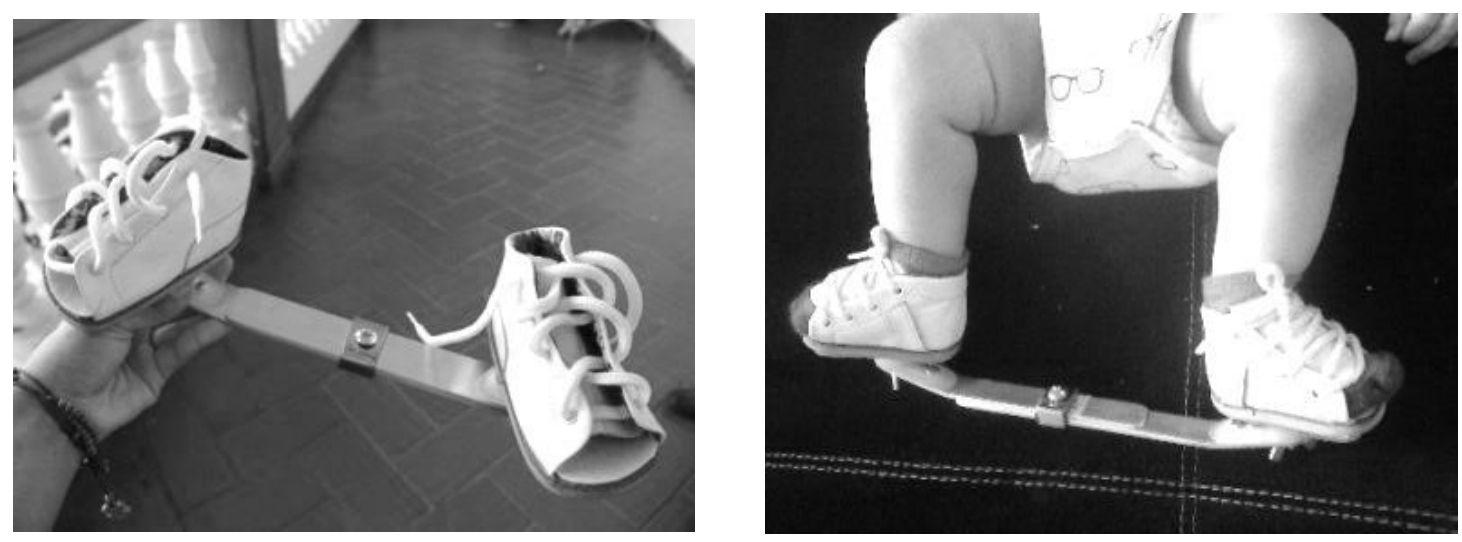

Figura 2. Férula de Dennis Brown. Paciente usando la férula.

La nueva propuesta de diseño aquí presentada, surge debido a que después de terminado el tratamiento de Ponseti en los niños con pie equino varo, se detecta que existe una reaparición de la deformidad si no se utiliza la órtesis durante los tres meses recomendados (Thacker et al., 2005).

Teniendo en cuenta que los niños en etapa de crecimiento son muy activos, muchos de ellos rechazan su uso ya que les impide realizar de forma normal sus actividades diarias, como gatear o caminar, por esta razón muchos padres acceden a los deseos del niño, retiran la órtesis y de esta forma promueven una recidiva de la deformidad.

Thacker et al. (2005) reportaron en su estudio un 58\% de reaparición en pacientes que incumplían con el uso de la órtesis, en comparación con un o\% de recidiva en pacientes obedientes. En otro estudio se obtuvo una tasa de recaída del $30 \%$ por el no uso de la órtesis, lo cual fue atribuido según testimonio de los padres a la irritabilidad y rechazo del niño en crecimiento o a tener la órtesis puesta incluso a la hora de dormir (Hazem \& ElTayeby, 2012). Estos estudios indican que la órtesis que se ha estado utilizando hasta el momento no cumple por completo con las expectativas, sin embargo, no se han encontrado estudios que indiquen un rediseño de la misma, con el fin de disminuir el rechazo a su uso en niños que se encuentran en crecimiento. Otro de los problemas encontrados era que los bebés eran capaces de generar la suficiente fuerza al hacer el gesto de la patada, como para provocar un daño significativo a la férula y sus componentes.

Cuando no se logra corregir esta deformidad las consecuencias condicen a problemáticas físicas, fisiológicas y sociales para el paciente, su familia y la sociedad. El niño podría llegar a encerrarse en un ciclo de incapacidad, dependencia, desmoralización, depresión y discriminación. La incapacidad física es una de las principales causas de afección y pobreza en países en desarrollo, ya que al tener oportunidades reducidas de participación en la educación o la vida profesional presentan desventajas económicas y sociales, en detrimento de su participación en la sociedad (Paschoal et al., 2011). 


\section{Método}

\section{Participantes}

Diez padres y sus bebés de entre 3 y 12 meses de edad, cinco con pie equino varo atendidos en la Fundación Infantil Clínica Club Noel de Santiago de Cali (Colombia) y cinco sin la patología. 17 Padres de bebes con pie equino varo y profesionales especialistas en ortopedia que atienden la población, para la recolección de testimonios y entrevistas referentes a la problemática abordada.

\section{Instrumentos y materiales}

Laboratorio de análisis de marcha de la Universidad Autónoma de Occidente: 9 cámaras marca Bassler GigaByte Ethernet de alta resolución, Software $\mathrm{MAXPRO}^{\circledR}$ y MOKKA ${ }^{\circledR}$. Plataforma SHIMMER ${ }^{\circledR}$ (Shimmer, 2014), modulo acelerómetro, cinta métrica, goniómetro y dinamómetro. Software Matlab 2010, Software SolidWorks $2014^{\circledR}$, Software CES Edupack $2014^{\circledR}$.

Fabricación del prototipo: scaner Biosculpture 3D C, máquina de termo formado, 1 lámina de polipropileno de 4 mm, foami, acrílico, MDF 4 mm.

\section{Procedimiento}

Se hizo búsqueda de las férulas para pie equino varo actualmente disponibles en el mercado. Con el fin de determinar todos los problemas de diseño asociados al rechazo en el uso, se recopilaron testimonios (17) de padres de bebés que presentaban esta enfermedad y estaban usando la férula en ese momento, así como de profesionales. Los datos obtenidos de los testimonios de los padres, la revisión de la bibliografía y la entrevista al personal profesional fue analizada posteriormente bajo la metodología de Ulrich y Eppinger (2004).

Se procedió a realizar el diseño de concepto, que según la metodología de Ulrich y Eppinger (2004) consta de los siguientes pasos: Listado y clasificación de necesidades las cuales se obtienen de las encuestas a los usuarios, la revisión bibliográfica y la entrevista al personal profesional; jerarquización dichas necesidades agrupando las que tienen relación entre sí; ubicación en un diagrama denominado árbol de objetivos, el cual muestra las relaciones que hay en dicha jerarquización de una manera más clara; la matriz de comparación por pares, que da una clasificación cuantitativa a los objetivos; y el árbol de objetivos ponderado muestra esta clasificación, con lo cual se puede determinar qué objetivos serán abordados principalmente para el desarrollo del dispositivo.

Se realiza una matriz de qué - cómo, que relaciona los objetivos y lo que se requiere para cumplirlos; se realiza un diagrama llamada casa de la calidad QFD (Quality Function Deployment) en el cual se pondera tanto las necesidades como las especificaciones en las que finalmente se centrará el diseño; se realiza la matriz de necesidad - medidas para determinar las características que debe tener el diseño; se hace un análisis del producto existente en el mercado y de los valores objetivos que tienen el material, precio, etc. Finalmente se produce la generación del concepto.

Con este procedimiento se pudo determinar cuáles eran los objetivos que debía seguir el rediseño de la órtesis de una manera objetiva; estipular qué se debía hacer y cómo se iba a lograr; enfocar el diseño a dar respuesta a las necesidades identificadas; analizar las características más importantes de las férulas existentes en el mercado para establecer cuáles eran funcionales y efectivas, y cuales se debían cambiar; y finalmente, determinar los actores que iban a influir sobre el objeto y que funciones debía cumplir para lograr el objetivo establecido. 
Con base en el análisis biomecánico de las etapas del desarrollo locomotor de niños de 3 a 10 meses de edad, se procedió a realizar una toma de datos a través de la captura de movimiento de un bebe usando la férula y otra sin usarla, para determinar la diferencia en el gateo en estas dos situaciones e identificar las diferencias respecto al uso de la férula en este movimiento. Se ubicaron los marcadores siguiendo el protocolo Helen Hayes quitando el marcador del segundo metatarso

El estudio de movimiento con el uso de las cámaras permitió el registro mediante marcadores que se ubican en el paciente. La frecuencia de muestreo fue de $60 \mathrm{~Hz}$. Para la adquisición se usó el software MAXPRO ${ }^{\circledR}$ y el procesamiento se realizó por medio del software $\mathrm{MOKKA}^{\circledR}$. Cabe aclarar que para el estudio de movimiento en gateo no se tenía registros de ensayos anteriores ni su metodología (Montenegro, 2013).

El estudio de movimiento con el uso de las cámaras permitió el registro mediante marcadores que se ubican en el paciente. La frecuencia de muestreo fue de $60 \mathrm{~Hz}$. Para la adquisición se usó el software $\mathrm{MAXPRO}^{\circledR}$ y el procesamiento se realizó por medio del software MOKKA ${ }^{\circledR}$. Cabe aclarar que para el estudio de movimiento en gateo no se tenía registros de ensayos anteriores ni su metodología (Montenegro, 2013).

Se realizó la toma de datos de la fuerza con la que los bebes de las edades establecidas, realizaban el gesto de la patada, para obtener un valor con el que se pudiera escoger el material. Se tomó la información a 10 voluntarios, 5 bebés con pie equino varo y 5 bebés sin la patología, los cuales sirvieron de control, el promedio de las edades fue de 6.5 meses $(\mathrm{SD}=3.27)$, el peso $7.33 \mathrm{~kg}(\mathrm{SD}=1.11)$ y la talla $66.3 \mathrm{~cm}(\mathrm{SD}=4.62)$.

Los bebés se colocaron en posición supina sobre una camilla, y mediante un sensor SHIMMER ${ }^{\circledR}$, se registró la aceleración de la pierna de los bebés en el momento en que el tobillo hace impacto con la superficie. Se usó un sensor SHIM$\mathrm{MER}^{\circledR}$ con el módulo de acelerómetro, el cual se sujetó a cada uno de los pies del bebe a la altura de los tobillos (Ver Figura 3). Las dimensiones del sensor son $5 \times 3 \times 1.7 \mathrm{~cm}$ (largo x ancho x alto) y tiene un peso total de $24.32 \mathrm{~g}$ (Shimmer, 2014).

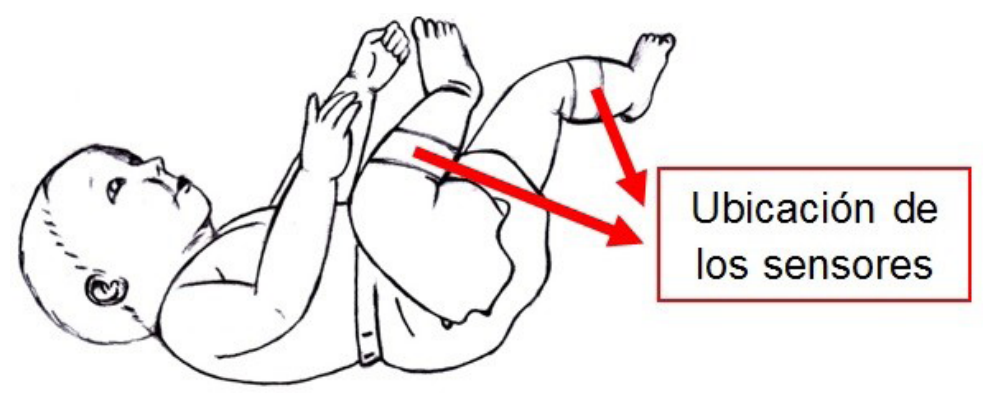

Figura 3. Ubicación de los sensores en el bebé, posición supina.

Mediante la señal de aceleración se calculó la velocidad y a partir de ella, usando los datos inerciales de los segmentos corporales involucrados, se calculó la magnitud y dirección de la fuerza de impacto de la patada. La revisión de los datos de acelerometría permitió concluir que la patada se desarrolló en un plano definido por los ejes X y Y, por lo cual se descartaron los datos en la dirección $\mathrm{Z}$.

$$
\begin{aligned}
& \int_{t 1}^{t 2} f=m \cdot\left(V_{2}-V_{1}\right) \\
& F r=\sqrt{F x^{2}+F y^{2}}
\end{aligned}
$$

Donde Fr corresponde a la fuerza resultante. 
Se evaluó igualmente la fuerza generada por la plantiflexion y la aducción, ya que estos son los movimientos propios de la recidiva. Estas fuerzas fueron tomadas con un dinamómetro de $10 \mathrm{~N}$ atado a la parte distal del pie. Se puso el pie recto, es decir, formando 90 grados con la pierna, posición en la cual se estableció el cero del dinamómetro. Cuando el bebé realizó la plantiflexión se registró el valor de la fuerza.

Igualmente para medir la fuerza de aducción, se puso el pie formando 90 grados, y se esperó que el bebé realice el movimiento correspondiente (Ver figura 4). Para lograr ambos movimientos, se estimuló el pie realizando una fuerza leve contraria al movimiento requerido, así el bebé tiende a realizar una fuerza en oposición.
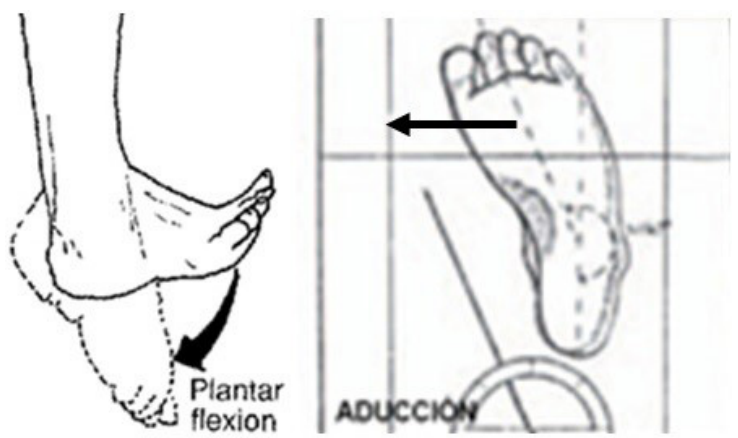

Figura 4. Dirección fuerza plantiflexión y aducción.

Se generaron 6 modelos de alternativas de solución diferentes los cuales fueron evaluados según su costo, cosmesis, confort y control; de esta evaluación se escogió la alternativa con el mejor puntaje.

La alternativa escogida fue modelada por medio del software SolidWorks@, con el cual también se evaluó el desempeño de la órtesis teniendo en cuenta las fuerzas involucradas y el estudio biomecánico anteriormente descrito.

Para la selección del material se usó el procedimiento descrito por Ashby, usando como criterio de selección la relación $\frac{E^{1 / 3}}{\rho}$ la cual permite seleccionar materiales para aplicaciones en donde es necesaria una alta rigidez y un mínimo peso. Por lo tanto se buscó un material con un alto módulo de Young (eje vertical) y una baja densidad (eje horizontal) (Ver Figura 5), por esto que se decidió escoger los polímeros de ingeniería, dentro de los cuales el polipropileno fue la mejor opción.

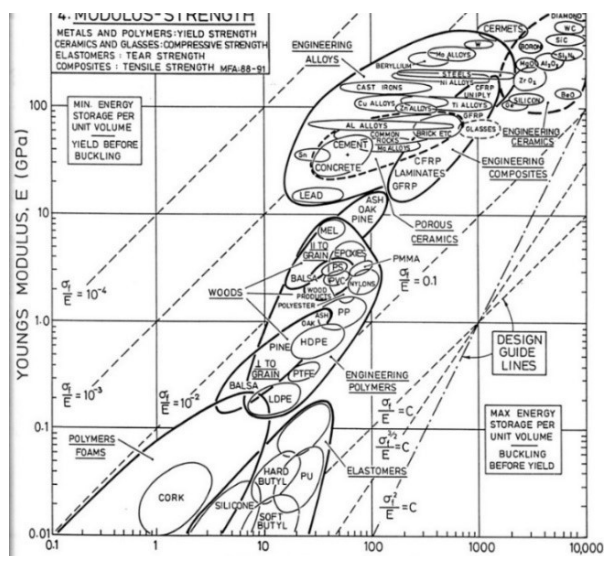

Figura 5. Módulo de Young vs Fuerza (carga). 
Se procedió a fabricar el prototipo alfa del modelo escogido y evaluado previamente en la plataforma virtual. Se hizo escaneo 3D del pie de uno de los bebés mediante un scaner Biosculpture 3D C y se fabricó el molde usando láminas de MDF de $4 \mathrm{~mm}$ apiladas y cortadas mediante cortadora laser, finalmente, mediante la técnica de termo formado se fabricó el prototipo con lámina de polipropileno de $3 \mathrm{~mm}$. El interior del modelo se recubrió con un material suave para evitar incomodidades (ver figura 6).
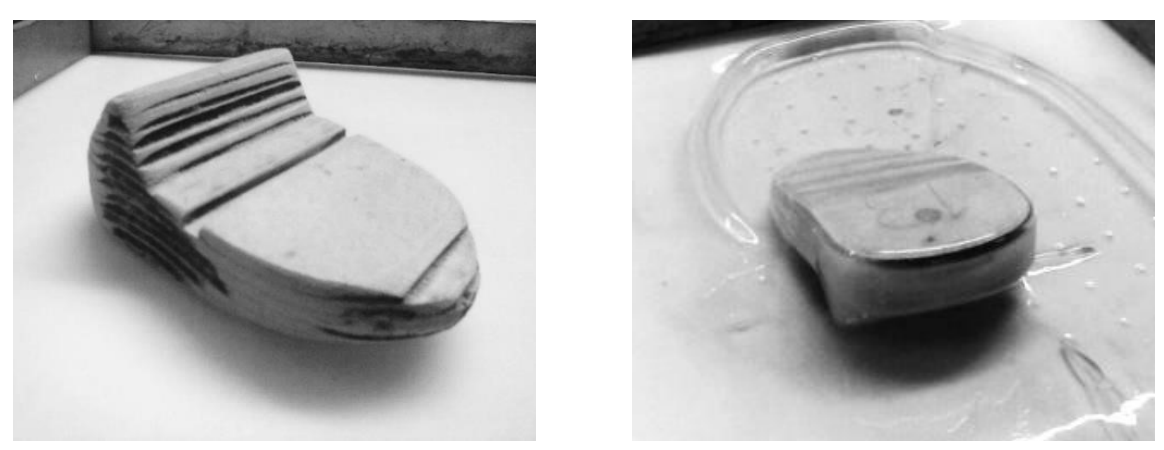

Figura 6. Molde en MDF de una pieza de la propuesta de diseño, termo formado del polipropileno sobre el molde.

Finalmente se hicieron pruebas de tracción, flexión e impacto al material escogido, para verificar que el material cumplía con todos las necesidades requeridas. Los resultados de estas pruebas, comparadas con los valores teóricos obtenidos del software CES Edupack $@$, se muestran en la tabla 1.

Tabla 1.

Comparación información CES Edupack 2014 y pruebas de laboratorio.

\begin{tabular}{clccc}
\hline & CES Edupack & & Pruebas & unidades \\
\hline Young's modulus & 1.02 & 1.04 & 0.961 & $\mathrm{GPa}$ \\
Yield strength (elastic limit) & 21 & 24.3 & 28.912 & $\mathrm{MPa}$ \\
Tensile strength & 19.3 & 20.3 & 22.529 & $\mathrm{MPa}$ \\
Elongation & 7.79 & 54.3 & 38.112 & $\mathrm{GPa}$ \\
Compressive modulus & 1.02 & 1.04 & - & $\mathrm{MPa}$ \\
Compressive strength & 37 & 38.8 & - & $\mathrm{MPa}$ \\
Flexural strength & 24.6 & 31.9 & 20.369 & $\mathrm{GPa}$ \\
(modulus of rupture) & & 0.376 & 0.2 & \\
Shear modulus & 0.367 & & & \\
\hline
\end{tabular}

Por último, se realizaron pruebas funcionales al dispositivo final para verificar el cumplimiento de la corrección del pie en abducción y comprobar que la dorsiflexión llegue hasta $60^{\circ}$ de flexión de tobillo. Se probó el prototipo colocándolo sobre el pie de un voluntario que cumplía con la edad de la población de estudio, y se verificó la correcta ubicación del pie y el gateo con libertad. 


\section{Resultados}

De acuerdo con la recopilación de información se determinó que el rango de edad en la que los niños empiezan a usar la férula hacia los 304 meses y presentan mayor dificultad con la férula cuando están entre los 6 meses y 1 año dado que el bebé tiene la capacidad de cambiar de posición de supino a prono y porque en esta etapa está empezando a gatear. En este sentido, la dificultad en el uso de la férula es causada por la barra que une los dos zapatos.

El movimiento de gateo con y sin férula son distintos debido a la restricción de la separación de los pies. Normalmente durante el gateo, la progresión del movimiento se realiza por medio de las rodillas. Con la férula, el bebé tiene una restricción del avance lo cual compensa mediante la rotación de cadera, aumentando el gasto energético y consecuentemente, se reduce la velocidad de avance. Se observa además como un indicador cualitativo del aumento del gasto energético, que el bebé hace breves pausas antes de continuar con el gateo. La velocidad promedio de gateo sin la férula es de $0.48 \mathrm{~m} / \mathrm{s}$ (distancia: $4.8 \mathrm{~m}$ - tiempo: $10 \mathrm{~s}$ ), y con la férula se reduce a $0.37 \mathrm{~m} / \mathrm{s}$.

Para el análisis de movimiento se usó como marcador de referencia el marcador del Sacro (ver Figura 7), debido a que la línea de desplazamiento en el tiempo descrita por este marcador, en el plano horizontal, evidencia claramente la diferencia del gateo con y sin férula, resaltándose el aumento de amplitud de la curva descrita por el marcador, lo cual representa un incremento en la rotación pélvica del bebé durante el gateo con férula. Los demás marcadores describen el movimiento de las extremidades, pero no se evidencian claramente características diferenciadoras concluyentes. Se entiende que el análisis de movimiento sin la férula representa el movimiento que realizaría el paciente con la nueva propuesta de diseño.

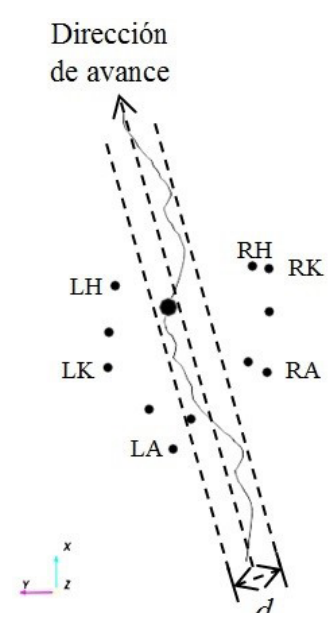

(a)

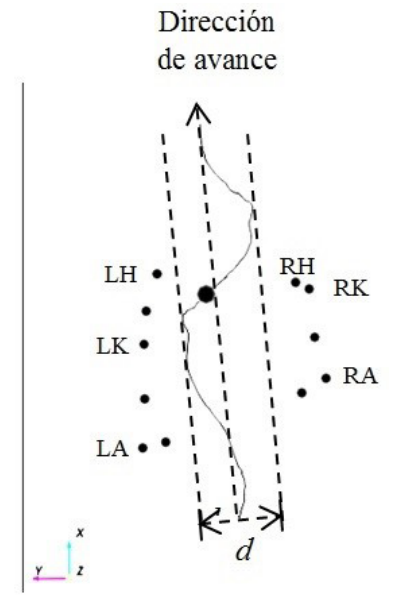

(b)

Figura 7. Vista coronal de la trayectoria descrita por el marcador ubicado en Sacrum (Sacro), durante el gateo. LH, LK, LA marcadores de la cadera, rodilla y tobillo izquierdo, respectivamente. RH, RK, RA marcadores del miembro derecho. (a) Desplazamiento sin férula (b) Desplazamiento con férula. Donde d es la amplitud de desplazamiento del sacro. Se observa un incremento en la amplitud de la trayectoria del sacro lo cual evidencia un aumento de la rotación pélvica.

Del estudio de fuerzas se determinó que la fuerza generada en plantiflexión fue aproximadamente $4 \mathrm{~N}$ y la fuerza generada en aducción fue de $2 \mathrm{~N}$. La velocidad calculada debida a la aceleración del gesto de la patada fue de: $47.4169 \mathrm{~m} / \mathrm{s}$. 
Cabe aclarar que estas fuerzas son solo una estimación, y que los recursos y la técnica de medida se pueden cambiar. Las fuerzas tomadas son activas, en cambio las fuerzas que tiene que soportar la férula debida a la recidiva son pasivas, generan una presión constante y por un tiempo prolongado.

A partir de los resultados obtenidos, se pudo determinar qué ventajas y restricciones tiene el nuevo diseño con respecto a los objetivos principales.

\section{Tabla 2.}

Ventajas y restricciones de la propuesta de diseño.

\begin{tabular}{|c|c|c|}
\hline criterios & ventajas & Restricciones \\
\hline corrección del pie & $\begin{array}{l}\text { Mantiene la corrección del pie, por medio } \\
\text { de la elongación de los tendones, dada la su for- } \\
\text { ma, y evita que la fuerza generada por el retroceso } \\
\text { elástico del varo, genere recidiva. }\end{array}$ & $\begin{array}{l}\text { Se debe estudiar los posibles puntos de } \\
\text { presión que pueda generar la forma y materia- } \\
\text { les, con el fin de evitar una lesión en la piel o en } \\
\text { alguna estructura a largo plazo. }\end{array}$ \\
\hline ventilación & $\begin{array}{l}\text { La forma de la férula, mantiene la parte su- } \\
\text { perior de la pantorrilla y del pie libre, para evitar } \\
\text { exceso de calor. }\end{array}$ & $\begin{array}{l}\text { Los materiales pueden producir calenta- } \\
\text { miento por la generación prolongada de fricción. }\end{array}$ \\
\hline $\begin{array}{l}\text { finales y bordes } \\
\text { redondeados }\end{array}$ & $\begin{array}{l}\text { Cuenta con finales y bordes redondeados } \\
\text { para procurar la protección del usuario. }\end{array}$ & $\begin{array}{l}\text { Puede que el sistema de engranaje cause } \\
\text { alguna lesión. }\end{array}$ \\
\hline \multirow[t]{2}{*}{ ergonómico } & $\begin{array}{l}\text { El diseño de la férula se adapta al usuario, } \\
\text { dado que la forma está pensada y fabricada si- } \\
\text { guiendo las medidas de las extremidades involu- } \\
\text { cradas en la patología. }\end{array}$ & $\begin{array}{l}\text { El sistema de engranaje que evita la recidi- } \\
\text { va, puede ser muy engorroso al momento en el } \\
\text { que el bebé quiera aprender a pararse. }\end{array}$ \\
\hline & $\begin{array}{l}\text { Permite la generación del pataleo alternado } \\
\text { y un mejor desplazamiento en gateo. }\end{array}$ & \\
\hline liviano & $\begin{array}{l}\text { Los materiales de construcción de la férula } \\
\text { y del sistema de engranaje, son livianos ya que } \\
\text { pesan 104.63g. }\end{array}$ & \\
\hline portátil & $\begin{array}{l}\text { Al ser una férula de tamaño pequeño es } \\
\text { muy fácil de cargar y transportar. }\end{array}$ & \\
\hline fácil de reparar & $\begin{array}{l}\text { Al no tener muchas partes de ensamble y ser fa- } \\
\text { bricada con materiales comerciales, es fácil de reparar. }\end{array}$ & \\
\hline
\end{tabular}

Se pueden determinar de igual manera, las diferencias que hay entre el diseño que se encuentra en el mercado y el diseño propuesto. Se observa que el objetivo de la férula en cuanto a la ubicación del pie en posición de tratamiento se conserva. Las grandes diferencias están en el uso, ya que esta propuesta puede ser tanto para la patología bilateral como para la unilateral; y el precio, siendo este uno de los principales factores que influyen en la elección para la adquisición de esta férula. 
Tabla 3.

Comparación: Férula del mercado y la propuesta de diseño.

\begin{tabular}{|c|c|c|}
\hline $\begin{array}{c}\text { Criterios } \\
\text { Usos }\end{array}$ & $\begin{array}{c}\text { Férula mercado } \\
\text { Bilateral }\end{array}$ & $\begin{array}{c}\text { Propuesta de diseño } \\
\text { Unilateral }\end{array}$ \\
\hline Función & $\begin{array}{l}\text { Fortalece el músculo gastrosóleo } \\
\text { La bota y la curvatura de la barra permiten } \\
\text { la dorsiflexión del tobillo. }\end{array}$ & $\begin{array}{l}\text { Pataleo más natural, permite el movi } \\
\text { miento de las piernas individualmente. }\end{array}$ \\
\hline Materiales & $\begin{array}{l}\text { Zapatos de cuero, suela de goma, uniones de } \\
\text { termoplástico y barra de aluminio. } \\
\text { Cordones de tela y correa de cuero. }\end{array}$ & $\begin{array}{l}\text { Pataleo más natural, permite el movi } \\
\text { miento de las piernas individualmente. }\end{array}$ \\
\hline Medidas & $\begin{array}{l}\text { - } \text { diámetro del tobillo. } \\
\text { - longitud del empeine. } \\
\text { - longitud del pie. } \\
\text { longitud de los hombros. }\end{array}$ & $\begin{array}{l}\text { - diámetro tobillo. } \\
\text { - lámetro rodilla. } \\
\text { - longitud del pie. } \\
\text { - peso. } \\
\text { - talla. }\end{array}$ \\
\hline Número de ensambles & $\begin{array}{l}\text { - barra de metal. (1) } \\
\text { - } \text { mariposas de ajuste. (2) } \\
\text { - correa de cuero. (2) } \\
\text { total: } 7 \text { (ver Figura 9) }\end{array}$ & $\begin{array}{l}\text { estribo - pantorrilla. }(4) \\
\text { total: } 4 .\end{array}$ \\
\hline Peso & $\begin{array}{c}304.07 \mathrm{~g} \\
(2 \text { zapatos y barra) }\end{array}$ & $\begin{array}{c}104.63 \mathrm{~g}(\mathrm{c} / \mathrm{u} \text { férula) } \\
\text { total: } 209.26 \mathrm{~g}\end{array}$ \\
\hline Precio (CO) & $80000-100000$ & $30000-40000$ \\
\hline
\end{tabular}

\section{Discusión}

Cuando se recogió la información de los padres de los pacientes y los especialistas en el área de ortopedia, en primera instancia se determinó como principales necesidades diseñar la férula conforme al movimiento del gateo y al movimiento de pataleo que impedía su descanso por las noches. Con el fin de traducir las necesidades de los usuarios a cantidades medibles y guiar de manera objetiva el diseño del producto, se realizó la matriz QFD. Las necesidades de diseño que se destacaron fueron: la corrección del pie con un $27 \%$ siendo la principal función de la férula, que sea liviano y de fácil adaptación con un resultado del 17\%, que afecta al usuario directo, al igual que el que sea ergonómico. Otra necesidad importante a satisfacer fue la de fácil de poner, la cual afecta al usuario indirecto (padres de los pacientes).

Otro factor que surgió a medida que se tomaron las pruebas y que se pudo observar el comportamiento de los pacientes, fue que algunos alcanzaban la edad en la que pueden pararse con ayuda de un apoyo externo, lo que se convierte en otra necesidad (ver Figura 8). Debido al alcance de este proyecto se propone continuar con el rediseño, evaluando esta nueva necesidad. 

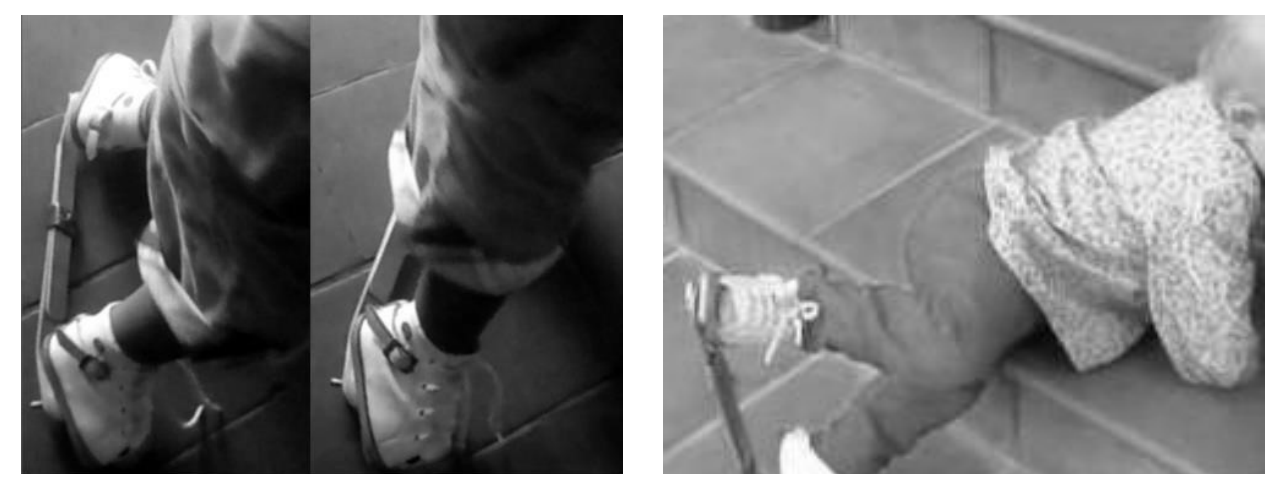

Figura 8. Restricciones de la férula del mercado: bipedestación y gateo.

Unas de las dificultades que se pudieron recopilar mediante las entrevistas y testimonio de los padres de los pacientes, y que está fuera del contexto de la funcionalidad de la férula pero que también puede provocar que el paciente tenga recidiva, es la efectividad de las Empresa Prestadora del Servicio de Salud al autorizar la colocación del siguiente yeso o la autorización de la cirugía. Al igual que la entrega tardía de la férula por parte del técnico que las elabora. Estas dificultades generan una prolongación en el tiempo del tratamiento, por lo que el pie del paciente tiende a volverse rígido y retornar a su forma patológica original, e incluso tienen que tomar el tratamiento desde el principio.

En este estudio se pudo determinar según los testimonios y encuestas que el 30\% de los padres están inconformes con el peso de la férula, en segundo lugar, la incomodidad que supone el uso representa un $26 \%$. El $22 \%$ de los padres concuerdan en que el material no es el adecuado, limita la movilidad tanto en el gateo como para la bipedestación y que el sistema de ajuste no permite que el pie permanezca en el zapato. Solo el 13\% de los padres manifestaron inconvenientes con la EPS en tanto que un solo padre manifestó que su hijo se pone triste con el uso de la férula.

La información que manejan los especialistas, los técnicos que hacen la férula y los padres de bebés con esta patología es incompleta. Se pudo observar fallas en la comunicación entre estos tres agentes, que genera de igual manera una falla en la continuidad del tratamiento, lo que afecta en última instancia al usuario principal de la férula.

Dentro de las necesidades que se pudieron determinar a partir de las pruebas realizadas, se tomó en cuenta solo el gateo del bebé, ya que fue muy difícil poder ver durante el estudio, el comportamiento del bebé mientras dormía, esto debido a que los horarios de descanso a esa edad no son regulares. Otro factor fue que las madres preferían que los niños durmieran durante el día sin la férula.

Los inconvenientes encontrados en las órtesis utilizadas actualmente y que están disponibles para su accesibilidad, para el tratamiento de pie equino varo congénito, son entre otros: la imposibilidad de pataleo alternado, dificultad para cambiar de posición de supino a prono, alteración de patrón de gateo y dificultad para pararse.

A partir de las pruebas biomecánicas que muestran el patrón de gateo de un paciente de pie zambo con y sin férula, se pudo determinar que hay una alteración, ya que se observa que el movimiento de rotación de cadera es más pronunciado cuando se realiza el gateo con la férula, generando así una compensación para lograr el avance, pero no es comparable con ningún estudio realizado anteriormente debido a que la locomoción en gateo no es considerado un movimiento funcional. Se recomienda hacer un estudio más profundo sobre la locomoción de los bebés de la edad de la población de este proyecto, para entender cómo posiblemente se puede alterar la marcha en el futuro, y así poder corregirla desde antes de que se presente. 
Los materiales y métodos usados en este proyecto, para realizar las mediciones correspondientes a las fuerzas y angulaciones dadas por las estructuras fisiológicas que generan la patología, pueden variar conforme se encuentre un método más efectivo o que produzca resultados más precisos. Se recomienda el estudio de métodos más eficientes para la toma de estas medidas.

Por medio del diseño del prototipo con la ayuda de una plataforma virtual se pudo visualizar de manera más efectiva las medidas y la forma que tendría el modelo, poniendo en contexto más real la eficiencia del diseño. Las limitaciones de este proceso es que no se puedan simular las pruebas en repetitividad, por lo que se hace muy difícil determinar un posible fallo en la férula por desgaste o fatiga en el tiempo.

La evaluación del desempeño del prototipo virtual con el análisis estático y dinámico, pudo determinar si era posible que el modelo real fuera a fallar o no, tanto en el material como con las partes móviles. A partir de esto se pudieron hacer modificaciones en el diseño antes de fabricarlo, generando una efectividad en el tiempo, en el proceso de elaboración y en el uso del material.

De acuerdo al alcance de este proyecto se puede determinar que es probable que se reduzca la formación de recidiva en pacientes con pie equino varo congénito, al reducir los factores determinantes propios de la férula que actualmente está en el mercado, que propiciaban el mal uso de dicha férula en el tratamiento, y por consiguiente el rechazo al uso por parte de los usuarios principales. Igualmente se pudo dar satisfacción a las opiniones y sugerencias que tenían los padres de los bebes.

Es importante que se le dé continuidad al estudio de este y otros diseños de férula para la corrección de esta patología, con el fin de encontrar ventajas y restricciones al momento de ponerlas a prueba con pacientes reales.

\section{Conflicto de Intereses}

Los autores declaran no presentar ninguna relación de interés comercial o personal dentro del marco de la investigación que condujo a la producción del manuscrito.

\section{Colaboraciones}

Todos los autores han contribuido intelectualmente en la elaboración del documento. 


\section{Referencias}

Hazem, M., \& ElTayeby, M. (2012). Multiple tenotomies after Ponseti Method for Management of Severe Rigid Clubfoot. The Journal of Foot \& Ankle Surgery, 51(2), 156-160.

Montenegro, M. (2013). Instructivo para el desarrollo del análisis biomecánico en el laboratorio de marcha de la fundación clínica infantil club Noel. Santiago de Cali, Colombia: Fundación Clínica Infantil Club Noel.

Pachajoa, H., Ariza, Y., Isaza, C., \& Méndez, F. (2011). Defectos congénitos mayores en un hospital de tercer nivel en Cali, Colombia 2004 - 2008. Revista de salud pública, 13(1), 152 - 162. Recuperado de: http://www.scielosp.org/pdf/ rsap/v13n1/v13n1a13.pdf

Paschoal, M., Rodríguez, J. C., Shiavom, P., Lourenco, A., Tedesco, A. P., Ferreira, L. A.,..., \& Renno, M. H. (2011). Brasil: A national program to eradicate neglected clubfoot-preliminary results. The Iowa Orthopaedic Journal. 31(1), 43-8. Recuperado de: http://www.ncbi.nlm.nih.gov/pmc/articles/PMC3215112/

Shimmer. (2014). 9DOF Calibration Application UserManual Rev 2.4a. Accelerometer coordinate system. Boston, USA.

Thacker, M. M., Scher, D. M., Sala, D. A., Van Bosse, H. J., Feldman, D. S., \& Lehman W. B. (2005). Use of the foot abduction orthosis following Ponseti casts: is it essential? Journal of Pediatric Orthopaedics, 25(2), 225-228. Recuperado de: http://www.researchgate.net/publication/8014290_Use_of_the_Foot_Abduction_Orthosis_Following_Ponseti_Casts_Is_It_Essential

Ulrich, K., \& Eppinger, S. D. (2004). Diseño y Desarrollo de Productos: Enfoque Multidisciplinario. (3ra ed). México D.F, México: McGraw-Hill

Zarante, I., Franco, L., López, C., \& Fernández, N. (2010). Frecuencia de malformaciones congénitas: evaluación y pronóstico de 52744 nacimientos en tres ciudades colombianas. Biomédica, 30(1), 65 -71. Recuperado de: http://www. redalyc.org/articulo.oa?id=84312378009

\section{Notas}

Artículo derivado de la investigación titulada "Rediseño de la órtesis para corrección post-tratamiento de pie equino varo congénito en niños en etapa de crecimiento en la ciudad de Santiago de Cali financiada con recursos propios.

La tecnología desarrollada es susceptible de protección mediante patente como modelo de utilidad por lo cual se ha obviado información que podría comprometer tal proceso. 
Información de autores:

\section{Oscar Iván Campo Salazar}

PhD Ingeniería eléctrica y electrónica

Docente Departamento de Automática y Electrónica. Universidad Autónoma de Occidente, Cali, Colombia oicampo@uao.edu.co

ORCID 0000-0002-5007-9613

\section{María Isabel Montenegro Muñoz}

Ingeniera Biomédica

Investigadora Universidad Autónoma de Occidente, Cali, Colombia

mimontenegro@uao.edu.co

ORCID 0000-0001-9336-6455 